\section{Editor-in-Chief \\ Barbara McLain - (retired Prof.) \\ University of Hawaii, USA}

The Israeli Journal of Aquaculture (IJA) is an interdisciplinary journal that is dedicated to sharing new research and tested applications of aquaculture

The IJA is devoted to scholarly articles for improved aquaculture practices and related industries

The IJA is a peer-reviewed, open-access, electronic journal

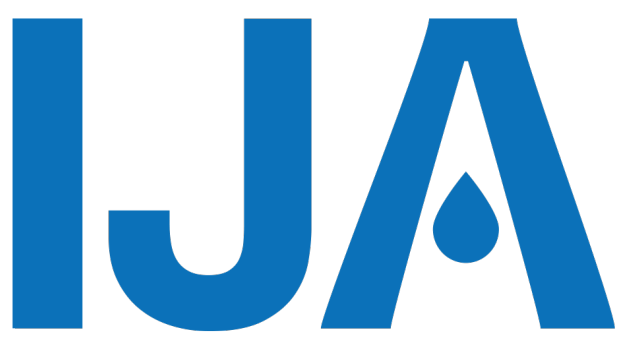

\section{The () Israeli Journal of Aquaculture}

An interdisciplinary online Open Access scientific journal

Published by the

\section{AquacultureHub}

A non-profit organization 501c3

http://www.aquaculturehub.org

in partnership with the

\section{University of Hawaii at Manoa} Library

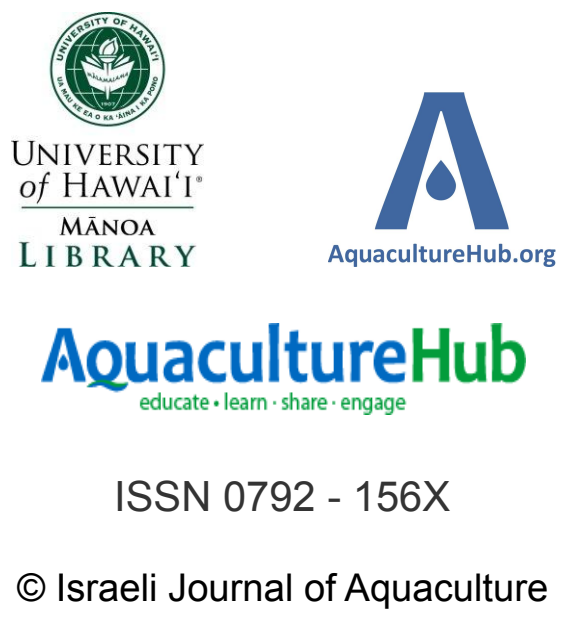




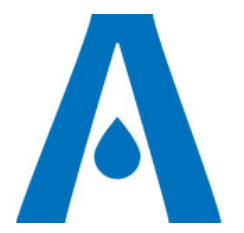

Produced by the AquacultureHub - nonprofit Foundation the IJA is an open-access, scientific journal, published on http://www. aquaculturehub.org

To read papers free of charge online at the above website Sale of IJA papers is strictly forbidden

\title{
Research progress on the cannibalistic behavior of Aquatic Animals and The Screening of Cannibalism-Preventing Shelters (Review)
}

\author{
Zhixing Su, Wenjun Xu, Hui Shi, Jie He* \\ Marine Fisheries Research Institute of Zhejiang province, Key Laboratory of \\ Marine Culture and Enhancement of Zhejiang Province, Zhoushan 316021, \\ China
}

\begin{abstract}
Cannibalism refers to maiming and killing among individuals of the conspecifics, and plays a key role in controlling animal population numbers and ensuring reproduction (i.e., eliminate the waste of food and space by weaker individuals). However, cannibalism in aquaculture directly reduces the survival rate and the production of farmed animals. Therefore, reduction in the incidence of cannibalism in farmed aquatic animals has been of great interest to the aquaculture industry. In this paper, we review factors affecting the cannibalistic behavior of aquatic animals and the research progress on the screening of shelters to prevent cannibalism. These shelters focus on the animals and their external environment. We also discuss prospects for future research. The purpose of this review is to provide a scientific reference for the study of cannibalistic behavior of aquatic animals and a theoretical basis for the development of cannibalism-preventing shelter technologies for aquatic animal farming.
\end{abstract}

Keywords: aquatic animals; cannibalism; shelter; progress

* Corresponding author: Jie He, E-mail address: he_0902@126.com Tel.: +8605802299820 


\section{Introduction}

Cannibalism refers to the maiming and killing among individuals, a phenomenon that is widespread in aquatic animals, especially among carnivorous species (Giles et al. 1986). Cannibalism, as a form of highly developed exclusive intraspecific consumption, is important to aquatic animals in terms of biological adaptability. Cannibalism enables certain species to alter their population density based on fluctuating environments and resources (Hua et al. 1998). Thus, cannibalism can determine intraspecific dominance and subordination, in which the winner obtain benefits that include resources, space, and mating rights (Hech et al. 1981; Li et al. 2013).

Cannibalism can effectively reduce the waste of space and food on weaker individuals to ensure the continuation of the population, thereby playing a promoting role in the evolution of the species. However, cannibalism also leads to the bodily injury, limb autotomy and even death in aquatic animals, posing a serious problem for aquaculture (He et al. 2016a; He et al. 2016b; Ge et al. 1986; Sotelano et al. 2016). In aquatic farming with the goal of high output, cannibalism leads to problems of low survival rates and economic deficits. Therefore, solving the negative impact of cannibalism in aquaculture is an important subject of study. Numerous studies have shown that installing shelters where farmed animals may hide, is an effective approach to mitigating cannibalism and improving the survival rate of the farmed animals (Hech et al. 1993; Johnston et al. 2006; Liu 2009; Xuan et al. 2015).

In this paper, we review the factors affecting the cannibalistic behavior of aquatic animals, and the progress on the application of shelters to prevent cannibalism in aquatic animals. We also discuss the prospects of future studies, with the goal of providing scientific reference for the study of cannibalistic behavior of aquatic animals. Finally, this student intends to provide a theoretical basis for the development of cannibalism-preventing shelter technologies for aquatic animal farming.

\section{Factors affecting cannibalistic behavior of aquatic animals}

At present, the factors causing cannibalistic of the conspecifics of aquatic organisms are an important subject for research. Scientists have found several factors that may promote cannibalistic behavior in aquatic species. These factors include: 1) individual size, 2) food abundance, 3) food type, 4) stocking density, 5) neurochemical factors, 6) light, 7) temperature and 8) water turbidity.

\section{Animal factors}

Kinship

Some fish exhibit specific kinship recognition, which can reduce cannibalistic incidence. This kinship is important for maintaining population size to survival of those species. Zaret et al. (1997) found that the Cichla ocellaris, peacock bass genus can avoid cannibalism by recognizing the eyes of other bass with alternating black and yellow colors - unique to its own species. Salmon effectively avoid cannibalism with a kinship identification mechanism that 
recognizes special chemical information (Wang et al. 2010). Liu et al. (2017) analyzed cannibalism and kinship of Japanese sea bass Lateolabrax japonicus juveniles through the molecular parent-child relationship information revealed by microsatellite DNA polymorphisms. Liu et al. (2017) showed that consanguineous (close relative) discrimination was present among juveniles, and cannibalistic incidences were reduced under appropriate feeding conditions. Cannibalism among juveniles of the southern catfish (Silurus meridionalis) $q \times$ catfish (Silurus asotus) o' hybrid is largely related to genotype, and juveniles of the same genotype show a low level of cannibalism. Additionally, the interaction between genotype and environment also plays a crucial role (Manley et al. 2015), Cichlid (Mckaye et al. 1976). For example, Cyprinodon macularius californiensis (Loiselle et al. 1983) display kinship recognition and rarely exhibit cannibalism among their offspring. Filial cannibalism is common, but less so if a parent recognizes their own eggs, as was found in the Poecilia species, where filial cannibalism was affected by the number of days post-hatching, in pupfish males and females differ (Loekle et al. 1982).

\section{Neurochemical factors}

The cannibalistic behavior of aquatic animals may be affected by neurochemical factors, such as 5-hydroxytryptamine, fluoxetine, octopamine, dopamine, and thyroid hormone. Hutchison et al. (1998) found that the cannibalistic behavior of thyroid hormone-treated Salmo trutta, Oncorhynchus mykiss and Onchorynkus masou, significantly decreased, indicating that an elevated level of thyroid hormone in fish plasma can effectively reduce cannibalism. Harlığlu et al. (2014) showed that adding supplemental tryptophan to feed can significantly reduce the aggressiveness of crayfish, and the higher the tryptophan, the higher the survival rate. Kania et al. (2012) found that decreased levels of 5-hydroxytryptamine in the brains of the fish Betta splendens intensified intraspecific infighting, whereas supplementing with different doses of 5-hydroxytryptophan almost completely eliminated the periodic cannibalism that occurs among males. In addition, Pedetta et al. (2010) examined the effects of regulation of 5 -hydroxytryptamine and octopamine on the cannibalistic behavior of Chasmagnathus convexus, and found that the behavior can be reversely regulated through 5-hydroxytryptamine and octopamine Thus increasing the 5-hydroxytryptamine content or decreasing the octopamine content can effectively mitigate cannibalism (Pedetta et al. 2010). These studies indicated that cannibalistic behavior of aquatic animals can be regulated by chemical factors, and the addition of exogenous neurochemical factors in the feed can effectively reduce the incidence of cannibalism.

\section{Parent-offspring adaptability}

Some animal parents eat their offspring, and this may be more common in aquatic animals (Manica, 2002). In general, cannibalism of offspring is detrimental to the species, but under some circumstances, it can be advantageous for eliminating weak individuals and conserving environmental resources. Dumont and Ali (2004) found that, when the population density of fairy shrimp Streptocephalus proboscideus reached a certain threshold, the newly hatched 
offspring were eaten by their parents, suggesting that if density of adults is sufficiently high, any immediately hatching eggs will be consumed without having reached molt stage. Cannibalism is implicitly, as the *adults* (not the juveniles) fed their own nauplii showed higher growth and reproductive output. However, this life history strategy is tightly linked to the existence of drought-surviving cysts and this allocation almost quantitatively recouped the loss of inclusive fitness to cannibalism. Wootton (1971) argued that even in the period that parental fish (sticklebacks) typically protect newly hatched offspring, when there is instance of food shortage, they consume a small part of offspring (stolen eggs were frequently not consumed) to supplement the nutrients that are capable of protecting the remaining offspring. This then ensures continued life until the next oviposition. Rohwer (1978) and Manica (2002) indicated that adult male sticklebacks regarded the consumption of egg as a courtship strategy. Parental common goby Pomatoschistus microps are capable of distinguishing eggs at different developmental stages and selectively cannibalizing eggs with low reproductive value to reduce the "net" cost of reproduction (Vallon \& Heubel 2016). In addition, parents can adjust the size and combination of their offspring by cannibalizing some offspring according to their environment (e.g., abundance and living space). For example, they remove sick or dead embryos from their reproductive organs to reduce the spread of disease, and also cannibalize offspring that are underdeveloped. This avoids providing additional energy for their care, and the energy saved by the parents' cannibalism can be used to care for the remaining offspring or for future reproductive activities (Frommen 2017). In natural environments, parents may cannibalize their offspring merely to ensure the continuation of the population. In addition, some research revealed that how the parents make the choice to cannibalize their offspring and identify those of low value remains by factors e.g. predator presence, oxygen levels and rival males etc. (Lissaker et al. 2003, Lissaker \& Kvarnemo 2006, Jones \& Reynolds 1999), but the exact reasons also need further explored.

\section{Size differences among individuals}

The effect of individual body size differences on cannibalism in aquatic animals has been extensively investigated. Body size differences have been shown to be an important factor affecting cannibalism. In the fry-rearing period, cannibalism is considerable among striped pufferfish Takifugu obscurus juvenile individuals with various body sizes under the same feeding conditions (Hua et al. 1998; Zhao \& Zhao 1999). Chen et al. (2003) found that when raised separately, kuruma prawn Penaeus japonicus individuals with different body lengths rarely exhibit cannibalism, but under mixed culture, those that are small are often cannibalized by those that are large. Under restricted feeding conditions, size differences among bluefin tuna juveniles led to cannibalism, and the larger the size difference, the more severe the mortality due to cannibalism (Ishibashi et al. 2013). Moreover, studies on red drum Sciaenops ocellatus, atlantic cod Gadus morhua, African catfish Clarias gariepinus, blue crab Callinectes sapidus, blue-swimmer crab Portunus pelagicus and southern king crab Lithodes santolla all confirmed that body size differences among juvenile individuals had a significant effect on their cannibalistic behavior (Liao \& Chang., 2002; Folkvord \& Ottera 1993; Folkvord 1991; Adamek 
et al. 2011; Marshall et al. 2005; Sotelano et al. 2012). Generally, cannibalism intensifies as the degree of heterogeneity in body size increases, and small individuals are often eaten by large individuals. Therefore, the rearing of individuals of specific aquatic animal species should incorporate segregation by body size during the farming process to mitigate cannibalism.

\section{Environmental factors}

Food abundance

Without body size differences, starvation will exacerbate cannibalism among aquatic animals. Under artificial feeding conditions, the cannibalism-derived loss rate of Asian seabass Barramundi juveniles was $1.08 \%$ per day, whereas in the test group that did not feed for $24 \mathrm{~h}$, the rate was $2.30 \%$ (Liu et al. 2017). When starvation for more than $12 \mathrm{~h}$, black rockfish Sebastes schlegelii juveniles exhibited a significant increase in cannibalism frequency (Xi et al. 2017). Ribeiro et al. (2015) found that under conditions of low feeding frequency, the competition for food was high, leading to heterogeneous growth, size differentiation, and ultimately cannibalism in barramundi Lates calcarifer juveniles. This is in contrast to high feeding frequency which resulted in homogenous growth and reduced cannibalism frequency in juveniles. Qin \& Li (2014) and Zhang (2006) found that feed frequency significantly affected cannibalism in Fenneropenaeus chinensis, and in a certain range, the lower the feeding frequency, the higher the rate of cannibalism. In addition, studies on juveniles of southern catfish Silurus meridionalis, pacific bluefin tuna Thunnus orientalis, and spotted seatrout Cynoscion nebulosus also indicated that feed abundance exerted a significant impact on cannibalistic behavior (Manley et al. 2015; Ishibashi et al. 2013; Zou et al. 2001). Therefore, under conditions of food shortage, larger individuals subsequently cannibalized smaller individuals, and the cannibalism frequency was positively related to the degree of food shortage. Thus, in aquaculture production, ensuring food abundance and increasing feeding frequency, can significantly reduce cannibalism incidence and improve the survival rate of farmed animals.

\section{Food type}

Food type also affects cannibalism rate among aquatic animals. Qin et al. (2014) found that shrimps fed with Artemia sinica showed more severe cannibalism than those fed with prawn crackers. A breeding experiment on Octopus ocellatus juveniles showed that cannibalism among those fed with live bait was significantly lower than among those fed with formulated feed (Wang et al. 2009). Ma et al. (2003) also found that different feed types significantly affected the cannibalistic behavior of catfish Silurus asotus juveniles, whereas Wang et al. (2008) found that feed types had no significant impact on the cannibalism among southern flounder Paralichthys lethostigma juveniles. Thus far, the effect of feed type on animal cannibalistic behavior has not been fully investigated as existing studies are limited to only a few animal species, and causal mechanisms have not been clearly established. 


\section{Population density}

The effect of population density on aquatic animal cannibalism has been extensively studied, and found to be an important factor affecting cannibalism. Giles et al. (1986) argued that, at a high population density, the competition of farmed fish for food, space, and dissolved oxygen intensifies. Thus, over time, the fish population becomes heterogeneous in body size, whereas the chance for predators and prey to encounter each other increases. This then further increases the frequency of cannibalism. For white shrimp Litopenaeus vannamei larvae density, when other conditions were identical, the density of larvae increased from 50 ind/L to 120 ind/L, and the average daily cannibalism incidence rate increased by $190 \%$ (Wang et al. 2015). Ribeiro et al. (2015) showed that under artificial farming conditions, as population density increased (regardless of feeding frequency), cannibalism among barramundi Lates calcarifer juveniles increased. Zou et al. (2001) also indicated that high population density intensified cannibalism among the southern catfish Silurus meridionalis larvae. Furthermore, cannibalistic behavior among the larvae of the Chinese white shrimp Fenneropenaeus chinensis and the subantarctic lithodid crabs Lithodes santolla increased with population densities, significantly reducing survival rate (Qin et al. 2014; Sotelano et al. 2016). In addition, Chen et al. (2003) found that, under different population densities, the cannibalistic behavior of kuruma prawn Penaeus japonicus differed significantly with population density and had a positive correlation. Chen et al. (2003) found that when the population density was above 100 ind/L, the frequency of cannibalistic behavior significantly decreased, indicating that population density had an effect on cannibalism, but was dependent on a density threshold. In summary, high population density often induces a high frequency of cannibalistic behavior, which is a problem urgently in need of a solution in the aquatic farming industry.

Physical and chemical factors of water

Temperature

At high temperatures, aquatic animals generally are more active, increasing their chance of encountering each other, and thus exacerbating cannibalism. In comparison, low temperature has a tranquilizing effect, making aquatic animals less active with slower movements, thereby decreasing their chance of encountering each other and lowering the cannibalism incidence. Li et al. (2001) found that the temperature adaptability of post-larval kuruma prawn Penaeus japonicus had a significant impact on cannibalistic behavior after artificial release. Additionally, they found that the reduction in the numbers of post-larval shrimp $5 \mathrm{~d}$ to $10 \mathrm{~d}$ after release was caused by cannibalism, which was mainly affected by temperature. At high water temperatures, the post-larval shrimp were active and showed strong feeding ability, resulting in severe cannibalism. At low water temperatures, shrimp were mostly static at the bottom of the water body, with little activity, infrequent feeding, and mild cannibalism. At $22{ }^{\circ} \mathrm{C}$, the survival rate of post-larval freshwater crayfish Cherax albidus reached 93\%, but this rate was only $57 \%$ at $28^{\circ} \mathrm{C}$, a significant difference (Verhoef \& Austin., 1998). In addition, Wang et al. (2009) found that temperature had a significant effect on cannibalism among Octopus ocellatus larvae; when the temperature was below $24^{\circ} \mathrm{C}$, where cannibalistic behavior was 
less frequent, and when the temperature was above $26^{\circ} \mathrm{C}$, daily cannibalism incidence significantly increased.

\section{Light}

Light was shown to have a significant effect on cannibalism in post-larval kuruma prawn Penaeus japonicus. These prawns are nocturnal, and under dark conditions when activity level is high, cannibalism increases, whereas intensive or long-term light conditions inhibit larval activity, decrease their encounter rate, and thus reduce cannibalism (Chen et al 2003). Xi et al. (2017) found that phototactic fish, such as black rockfish Sebastes schlegelii juveniles, tend to congregate at the bottom of water bodies during darkness, thereby increasing the chance of cannibalism, whereas light facilitates their dispersion, thereby reducing cannibalism ( $\mathrm{Li}$ et al. 2013). Adamek et al. (2011) also found that low-intensity light effectively suppresses cannibalism and improves the survival rate of African catfish Clarias gariepinus (photophobic speccies) larval juveniles. However, Pienaar et al. (1990) found that, under continuous light conditions, infighting and cannibalism among the photophobic Chinese catfish Clarias fuscus juveniles intensified even with abundant feed, indicating that different aquatic animal species exhibit different responses to light conditions (photophobism or phototaxis). Therefore, the light intensity should be adjusted according to each animal's phototropism to minimize light-induced cannibalistic behavior and improve the survival rate.

\section{Water turbidity}

The cannibalistic behavior of aquatic animals is also influenced by water turbidity. An appropriate increase in water turbidity can effectively lower the animal's visibility in the water body and hence provide an effective shelter for aquatic animals. Thus, turbidity reduces the animals' chances of seeing and encountering each other and facilitates hiding or escaping, thereby reducing cannibalism and territorial invasion (Pienaar et al. 1990). Li et al. (1992) found that black seabream Acanthopagrus schlegelii locate prey using vision, and an appropriate increase in water turbidity effectively reduced the chance of individuals finding each other, thus mitigating cannibalism. However, Liao et al. (2002) showed that although adding green alga to water increased the water turbidity, the cannibalistic behavior of Sciaenops ocellatus larvae was not significantly affected. Thus, the effects of water turbidity are different for each aquatic animal species. For aquatic animal species that rely on vision to seek prey, turbid water can affect the vision of the predator and thus reduce infighting and cannibalism; however, for those that seek prey without the aid of vision, water turbidity has been suggested to have little effect on cannibalistic behavior (Liao \& Chang. 2002,2010).

\section{Progress in creating shelters to prevent cannibalism among aquatic animals}

Cannibalism can effectively reduce the waste of space and food for disadvantaged individuals, ensure the continuity, and positively promote the evolution of the population. However, cannibalism negatively affects the mortality rate in aquaculture and lowers the income of farmers. Many field observations have proven that using appropriate shelters can reduce the 
frequency of intraspecies aggression in aquatic organisms and improve both the survival rate and yield. Researchers have begun to examine the effects of the construction, material, size and color of artificial shelters on aquatic animal cannibalism.

\section{Materials and configuration of aquatic shelters}

Numerous studies have been carried out on the material and configuration of shelters, and different aquatic species have exhibited significant differences in cannibalism under different shelter conditions. Heck et al. (1981) and Perkins-Visser et al. (1996) found that, under natural conditions, seagrass Zostera marina and shells can provide natural shelter for crabs and mitigate cannibalism. One research study found that installing a corrugated mesh shelter at the bottom of the cage can effectively improve the survival rate of mud crab Scylla serrata megalopae (David et al. 2007). The cannibalism rate of mud crab Scylla serrata in the presence of a bamboo shelter was $26 \%$ lower than that in the absence of a bamboo shelter (Mirera \& Moksnes 2013), and Triño et al. (1999) obtained similar results which using bamboo and seaweed as shelter materials. Liu (2017) compared the survival rates and growth conditions of red swamp crayfish Procambarus clarkia with or without shelters and showed that, in the early stage, the presence or absence of shelters had no significant effect on the survival rate. However, in a later stage of growth, the survival rate in the presence of shelters was significantly higher than that in the absence of shelters. In addition, the presence of shelters in the early stage effectively promoted the growth of the shrimp.

Del Mar et al. (2019) examined the effects of artificial green coconut fiber and natural seaweed shelter materials (both providing a large sanctuary space) for the growth and survival of spider crab Maja squinado megalopae. They found that the growth and survival rate of megalopae reared in the natural seaweed shelter were significantly higher than those of megalopae reared in the artificial coconut fiber shelter. Under laboratory conditions, He et al. (2017) constructed shelter structures using different materials and configurations (polyethylene mesh and plastic baskets). They found that shelters made with each material prevented cannibalism and enhanced survival rate among swimming crab Portunus trituberculatus juveniles. Although shelters made with plastic baskets were more effective, the outdoor breeding pond experiment indicated that the final survival rates were $30.3 \%$ and $45.0 \%$ for the megalopae growing with the polyethylene mesh and plastic basket shelter structures, respectively, amounting to 1.2 and 1.8 times, respectively, to that of the control group. Chen et al. (2009) constructed shelters with different materials (cotton, nylon mesh, PVC and bamboo), configurations ( 3 structures $\times 3$ sizes) and placement modes (horizontal and vertical) for megalopae and juvenile crabs of Scylla paramamosain, which were found to prefer no particular shelter material and to prefer vertically placed shelters. They showed significantly different preferences for particular configurations (shrub-typed, cave-typed, and angle-typed) and sizes ( $5 \mathrm{~mm}, 7 \mathrm{~mm}$ and $10 \mathrm{~mm}$ ), among which the shrub-type and small shelters were favored the most; the rates of their attachment to the small shrub-type shelters were $30.92 \%$ and $25.90 \%$, respectively, whereas those to the large shrub-typed shelters were $0.46 \%$ and $1.4 \%$, respectively. In a breeding experiment on post-larval western rock 
lobster Panulirus cygnus, Johnston et al. (2006) designed two kinds of shelters using a new type of rigid plastic mesh and bricks. They found that the survival rate of the post-larval lobsters with the rigid plastic mesh shelter was significantly higher than that of the post-larval lobsters with the brick shelter. Under identical stocking densities and diets, the survival rate of Scylla spp. megalopae with shelters made of mesh was significantly higher than that of megalopae with shelters made of blood clam Tegillarca granosa shells and sand (Shen et al. 2009).

A study (Song et al. 2012) of red swamp crayfish Procambarus clarkii also indicated that the broodstock survival rate was significantly affected by different shelter types. With a shelter made of 'waterweeds + asbestos tile + brick', the average broodstock survival rate was as high as $95.69 \%$; which was $9.6 \%$ higher than that for crayfish offered shelters made from 'waterweeds + asbestos tile + PVC pipe', and 3.5\% higher than that for crayfish offered the 'triangle-shaped shelter'. Between the shelters made of the macrophyte Elodea nuttallii and the benthic plant Myriophyllum verticillatum, the Chinese mitten crab Eriocheir sinensis preferred to moult and hide in the latter, which had a more complex structure (Zeng et al. 2018). Mirera and Moksnes (2015) constructed four types of shelters using seaweed, plastic rope, bamboo tubes and an open sand matrix for post-larval mud crab Scylla serrata and found that, in a small-scale rearing process, the shelters differed significantly in preventing cannibalism in the post-larval crabs. Compared to that of the open sand matrix shelter, the cannibalism-preventing rate of each of the other three shelter types was more than $50 \%$ higher. Gou et al. (2015) used three types of shelters, i.e., black or white PVC pipes with holes and the modified zhikong scallop Chlamys farreri rearing cage (a net for scallop feeding and/or trap for scallop capture), for Sebastes schlegelii larvae and found that, under natural light without shelter, the larvae were scattered in the shadowy areas in the corners of water tank and were inactive. After the shelter was installed, the larvae quickly moved towards the shelter, which exhibited a significant trapping effect (a powerful hidden behavior), and the modified zhikong scallop Chlamys farreri rearing cage was the most effective.

\section{Shelter size and color}

Shelter size and color should be compatible with the biological characteristics of the particular species to be sheltered. Only when the size and color align with the living habits and needs of aquatic animals is it possible for the artificial shelter to effectively prevent cannibalism among the animals. Qin et al. (2010) found that if the shelter was spacious enough to allow both large-sized and small-sized barramundi Lates calcarifer individuals to enter, the shelter instead exacerbated the cannibalistic behavior. For species that prefer a dark environment, such as Neoceratodus forsteri larvae, special attention should be paid to the fact that if the installed shelter is non-transparent or black, and too much light was applied, they may aggregate in the shelter for too long without leaving due to their strong preference for the dark environment, ultimately leading to death from hunger or hypoxia (Britz \& Penaar 2010). Furthermore, whether under natural light or shaded, black shelters had a higher efficiency in preventing cannibalism than white shelters for Sebastes schlegelii juveniles (Gou et al. 2015). 
Dong et al. (2010) installed shelters in six colors and found that sea cucumber Apostichopus japonicus larvae showed a color preference, with the attraction rate of the white shelter being higher than that of the green, red and blue shelters, whereas no significant difference existed among the white, yellow and black shelters. Moreover, giant freshwater prawn Macrobrachium rosenbergii fries favored red and black shelters, but disliked yellow, white, blue, brown, and green shelters (Li 1990). However, Ye et al. (1995) showed that shelters of different colors had no significant effect on the trapping and attachment of giant river prawn Macrobrachium rosenbergii and Macrobrachium nipponense fry.

\section{Shelter arrangement and quantity}

Improper shelter arrangement can impact the level of protection for aquatic animals. For example, summer flounder Paralichthys dentatus juveniles are benthic and usually active at the bottom of the water body. If the shelter is placed in the middle and upper layers of the water body, the juveniles are unable to hide in the shelter to avoid cannibalism (Francis et al. 2010). Chen et al. (2009) found that the megalopae and juvenile crabs of mud crab Scylla paramamosain favor hiding in vertically arranged shelters. Liu (2009) laid fiberglass tile shelters in an aquaculture pond and found that the specific structure of the shelter had a significant effect on the survival of Portunus trituberculatus. When the angles between the two shelters (shelters were both perpendicular to the bottom of the pond) were in the range of $30^{\circ}$ to $90^{\circ}$, the least cannibalism occurred. Moreover, the number of shelters should be adequate to meet the needs of all individuals; if the number of shelters is too small, individuals may compete for shelters, likely increasing the frequencies of cannibalism or territorial invasion (Hecht et al. 1993). Xi et al. (2017) installed triangular PVC panel shelters for rockfish Sebastes schlegelii juveniles and found that, when other conditions were constant, the higher the shelter density, the lower the cannibalism incidence among juveniles. Fang et al. (2011) showed that the number of bamboo shelters had no significant effect on the survival or growth of red swamp crayfish Procambarus clarkia, likely because the additional aquatic plants that were installed in the upper, middle and lower layers of the aquaculture water had already provided sanctuaries for these aquatic animals.

\section{Conclusion}

Cannibalism is one of the most important causes for mortality in aquatic animals. Currently, kin recognition, size difference among individuals, stocking density and feed abundance are believed to be the major factors leading to cannibalism. Many investigations have been undertaken on these factors, with suggestions of various mechanisms for reducing cannibalistic behavior. The occurrence of cannibalistic behavior differs between different species, and may be dominated by the biological and ecology of the animals, by environmental factors, or by the interaction between these factors. Cannibalistic behaviors also differ significantly at different developmental stages, such as the effect of molt stage on vulnerability to cannibalism, as well as the effect of size and swimming ability (hunting capacity) of growing larvae. Methods to prevent cannibalism in aquaculture species are still inadequate in commercial operations and require further study.

The introduction of shelters in breeding pools and tanks can effectively reduce the incidence of cannibalistic behaviors in aquatic animals. So far, the effects of shelter material, 
form, size and arrangement on the cannibalistic behavior of aquatic animals have been examined, and some progress has been made. However, many problems remain.in many aquatic animal species, shelters have been demonstrated to be effective in preventing cannibalistic behavior among the animals. However, the unstable configuration of the shelter may lead to negligible improvement of juvenile survival. Furthermore, some types of shelters are prohibitive due to difficult installation, inconvenient transportation, or cost. For example, a stone shelter is too heavy for installation and/or transportation (Wang et al, 2004) and tire shelters are expensive (Teng \& Chua, 1979). The types and materials of shelters reported so far are relatively simple and primarily include PVC pipes and polyethylene meshes, but they may not be suited for certain aquatic animal species. Moreover, the optimal number and arrangement of shelters in aquaculture production has not been adequately investigated, and the existing studies have been primarily conducted in a relatively safe, laboratory environment, whereas cannibalism-prevention effectiveness of these shelters has rarely been verified in pond production operations. Research for pond production shelters may have different results (Xuan et al,2015). At present, the use and success of cannibalism-preventing shelters in aquaculture is unresolved and requires further study.

\section{Acknowledgement}

This study was financially supported by the Natural Science project of Marine Fisheries Research Institute of Zhejiang province (No: 2019F304), and the scientific research start-up funding of Zhejiang Ocean University (No:22135010815).

\section{References}

Adamek J., Kamler E., Epler P., 2011. Uniform maternal age/size and light restrictions mitigate cannibalism in Clarias gariepinus larvae and juveniles reared under production-like controlled conditions. Aquacultural Engineering, 45(1), 13-19.

Britz P. J., Pienaar A. G., 2010. Laboratory experiments on the effect of light and cover on the behavior and growth of African catfish, Clarias gariepinus (Pisces: Clariidae). Proceedings of the Zoological Society of London, 227(1), 43-62.

Catacutan M. R., 2002. Growth and body composition of juvenile mud crab, Scylla serrata, fed different dietary protein and lipid levels and protein to energy ratios. Aquaculture, 208(1), 113-123.

Chen X. L., Li S. Q., Wang G, Z., 2008. Study on Relationship Between Cannibalism and Resource Availability/ Starvation of Mud Crab , Scylla paramamosain Megalopae. Journal of Xiamen University, 47(1), 99-103.

Chen X. L., Lin Q. W., Li S. Q., 2003. Observations and studies on the cannibalism among post larvae of Kuruma Prawn , Penaeus japonicus. J. Journal of Xiamen University, 42(3), 358-362.

Chen X. L., Zhang X. L., Li S. Q., 2009. Shelter preference of megalopae and first juvenile of mud crab, Scylla paramamosain (Estampador , 1949). Journal of Xiamen University, 48(4), 594-599 .

David L. M., Tom A., Beverley K., Ternt, L., 2007. Stocking density and artificial habitat influence stock structure and yield from intensive nursery systems for mud crabs Scylla serrata (Forsskal 1775). Aquaculture Research, 38(14), 1580-1587.

Dong G., Dong S., Wang, F., Tian, X., 2010. Effects of materials, incubation time and colors of artificial shelters on behavior of juvenile sea cucumber Apostichopus japonicus. Aquacultural Engineering, 43(1), 1-5.

Dumont H. J., Ali, A. J., 2004. Stage-Specific Cannibalism and Spontaneous Cyst Hatching in the Freshwater Fairy Shrimp Streptocephalus Proboscideus, Frauenfeld. Hydrobiologia, 524(1), 103-113.

Fang C. L., Zhou Z. Y., Wang, Z. Y., 2011. The effect of the quantity of shelterst and the 
setting of pond ridge on the reproduction performance of Procambarus clarkia. Jiangxi Fishery Science and Technology, 16(3), 16-18.

Francis A, W. J., Bengtson, D. A., 2010. Partitioning of fish and diet selection as methods for the reduction of cannibalism in Paralichthys dentatus larviculture. Journal of the World Aquaculture Society, 30(3), 302-310.

Frommen, J. G., 2017. Kin recognition and filial cannibalism in an amphibious fish. Behavioral Ecology \& Sociobiology, 71(10), 141.

Folkvord, A., 1991. Growth, survival and cannibalism of cod juveniles ( Gadus morhua ): effects of feed type, starvation and fish size. Aquaculture, 97(1), 41-59.

Folkvord A., Otter H., 1993. Effects of initial size distribution, day length, and feeding frequency growth, survival, and cannibalism in juvenile Atlantic cod Gadus morhua L'. Aquaculture, 114(3-4), 243-260.

Gardner C., Maguire G. B., 1998. Effect of photoperiod and light intensity on survival, development and cannibalism of larvae of the Australian giant crab Pseudocarcinus gigas, (Lamarck). Aquaculture, 165(1-2), 51-63.

Ge M. X., Xue, H. L., Zhang, W. X., 2000. A new method to improve the survival rate of juvenile Takifugu rubripes. Hebei Fisheries, 110(2), 34-35(in Chinese with English abstract).

Giles N., Wright R. M., Nord M. E., 1986. Cannibalism in pike fry, Esox lucius L.: some experiments with fry densities. Journal of Fish Biology, 29(1), 107-113.

Gou H. Y., Zhang X. M., Gao T. X., 2015. Effects of artificial shelters and feeding frequency on growth and behavior of juvenile Sebastes schlegelii. J. Journal of Fishery Sciences of China, 22(2), 319-331.

Harlıoğlu M. M., Harlıoğlu A. G., Yonar S. M., Duran, T. C., 2014. Effects of dietary I-tryptophan on the agonistic behavior, growth, and survival of freshwater crayfish Astacus leptodactylus, Eschscholtz. Aquaculture International, 22(2), 733-748.

He J., Gao, Y., Wang, W., 2016a. Limb autotomy patterns in the juvenile swimming crab (Portunus trituberculatus) in earth ponds. Aquaculture, 463, 189-192.

He J., Wu X., Cheng, Y., 2016b. Effects of limb autotomy on growth, feeding and regeneration in the juvenile Eriocheir sinensis. Aquaculture, 457, 79-84.

He J., Gao Y., Xu W. J., 2017. Effects of different shelters on the molting, growth and culture performance of Portunus trituberculatus. Aquaculture, 481, 133-139.

Hecht T., Pienaar A. G., 1993. A Review of Cannibalism and its Implications in Fish Larviculture. Journal of the World Aquaculture Society, 24(2), 246-261.

Heck K. L., Thoman, T. A., 1981. Experiments on predator-prey interactions in vegetated aquatic habitats. Journal of Experimental Marine Biology and Ecology, 53(2-3), 125-134.

Hua Y. Y., Chen, Y. F., Qian, L. F., 1998. Study on cannibalism of Takifugu obscurus. larvae. Acta Hydrobiologica Sinica, 22(2), 195-197(in Chinese with English abstract).

Hutchison M. J., Iwata, M., 1998. Effect of thyroxine on the decrease of aggressive behavior of four salmonids during the parr-smolt transformation. Aquaculture, 168(1), 169-175.

Ishibashi Y., Miki T., Sawada Y., Kurata, M., 2013. Effects of feeding conditions and size differences on aggressive behavior and cannibalism in the Pacific bluefin tuna Thunnus orientalis (Temminck and Schlegel) larvae. Aquaculture Research, 45(1), 45-53.

Johnston D., Melville-Smith R., Hendriks, B., Maguire, G. B., Phillips, B., 2006. Stocking density and shelter type for the optimal growth and survival of western rock lobster Panulirus cygnus (George). Aquaculture, 260(1-4), 0-127.

Jones, J. C., Reynolds, J. D., 1999. Costs of egg ventilation for male common gobies breeding in conditions of low dissolved oxygen. Animal Behavior, 57(1), 181.

Kania B. F., Mikoaj A., Wielgosz, G. M., 2012. Four-Week Fluoxetine (SSRI) Exposure Diminishes Aggressive Behavior of Male Siamese Fighting Fish . Journal of Behavioral \& Brain Science, 2(2), 185-190.

Li C. X., 1992. Observation on the turbidity of water and the phenomenon of cannibalism of Black seabream. Marine Fisheries.2, 58-59.

Li R. Y., Chen J. K., Jiang, H. L., 2001. Experiments on temperature suitable for postlarva of 
Penaeus japonicus. Fisheries Science, 20(3), 17-18.

Li W. J., 1990. Freshwater shrimp farming. Shang Hai Scientific \& Technical Publishers.

Li Y. Q., Sun, X., 2013. Agonistic behaviors of aquatic animals. Zoological Research, 34(3), 214-220.

Liao I. C., Chang, E. Y., 2002. Timing and Factors Affecting Cannibalism in Red Drum, Sciaenops ocellatus, Larvae in Captivity. Environmental Biology of Fishes, 63(2), 229-233.

Liao I. C., Chang, E., 2010. Role of sensory mechanisms in predatory feeding behavior of juvenile red drum Sciaenops ocellatus. Fisheries Science, 69(2), 317-322.

Liu K. Y., 2009. Effectof sand sediment and shelters on behavior, growth and survivorship of Portunus trituberculatus. D. Ocean University of China..

Liu Q., 2017. Procambarus clarkii response to two kinds of environmental heterogeneity and the selection of shelter. D. Nan Jing University.

Liu X., Xia, J., Pang, H., Yue, G., 2017. Who eats whom, when and why? Juvenile cannibalism in fish Asian seabass. Aquaculture \& Fisheries, 2(1), 1-9.

Lissåker M., Kvarnemo C., Svensson, O., 2003.Effects of a low oxygen environment on parental effort and filial cannibalism in the male sand goby, Pomatoschistus minutus. Behavioral Ecology, 14(3), 374-381.

Lissåker M., Kvarnemo, C., 2006. Ventilation or Nest Defense: Parental Care Trade-Offs in a Fish with Male Care. Behavioral Ecology \& Sociobiology, 60(6), 864-873.

Loekle D. M., Madison, D. M., Christian, J. J., 1982. Time dependency and kin recognition of cannibalistic behavior among poeciliid fishes. Behavioral \& Neural Biology, 35(3), 315-318. Loiselle P. V., 1983. Filial cannibalism and egg recognition by males of the primitively custodial teleost Cyprinodon macularius californiensis, girard (Atherinomorpha: Cyprinodontidae). Ethology \& Sociobiology, 4(1), 1-9.

Luppi T. A., Spivak E. D., Anger, K., 2001. Experimental studies on predation and cannibalism of the settlers of Chasmagnathus granulata and Cyrtograpsus angulatus(Brachyura: Grapsidae). Journal of Experimental Marine Biology \& Ecology, 265(1), 29-48.

Manley C. B., Rakocinski C. F., Lee, P. G., Blaylock R. B., 2015. Feeding frequency mediates aggression and cannibalism in larval hatchery-reared spotted seatrout, Cynoscion nebulosus. Aquaculture, 437, 155-160.

Maria del, M. G., Elena, P., Juana, D., 2019. Survival and growth of hatchery-reared Mediterranean spider crab juveniles, Maja squinado, under different rearing conditions. Aquaculture, 498, 37-43.

Marshall S., Warburton K., Paterson B., Mann, D., 2005. Cannibalism in juvenile blue-swimmer crabs Portunus pelagicus(Linnaeus, 1766): effects of body size, moult stage and refuge availability. Applied Animal Behavior Science, 90(1), 65-82.

Ma X. Z., Cui C. H., Wang, Z. Y., Jin X., 2003. Observation on Cannibalism and Study on the Feed of Silurus asotus. Fisheries Science, 22(1), 37-38.

Manica A., 2002. Alternative strategies for a father with a small brood: mate, cannibalise or care. Behavioral Ecology and Sociobiology, 51(4), 319-323.

Mckaye, K. R., Barlow G. W., 1976. Chemical Recognition of Young by the Midas Cichlid, Cichlasoma citrinellum. Copeia, 1976(2), 276-282.

Mirera O. D., Moksnes P. O., 2013. Cannibalistic interactions of juvenile mud crabs Scylla serrata: the effect of shelter and crab size. South African Journal of Marine Science, 35(4), 9. Mirera D. O., Moksnes P. O., 2015. Comparative performance of wild juvenile mud crab (Scylla serrata) in different culture systems in East Africa: effect of shelter, crab size and stocking density. Aquaculture International, 23(1), 155-173.

Moksnes P. O., Lipcius, R. N., Pihl L., Montfrans J. V., 1997. Cannibal-prey dynamics in young juveniles and postlarvae of the blue crab. Journal of Experimental Marine Biology \& Ecology, 215(2), 157-187.

Pedetta S., Kaczer L., Maldonado, H., 2010. Individual aggressiveness in the crab Chasmagnathus: Influence in fight outcome and modulation by serotonin and octopamine. 
Physiology \& Behavior, 101(4), 438-45.

Perkins-Visser E., Wolcott T. G., Wolcott D. L., 1996. Nursery role of seagrass beds: enhanced growth of juvenile blue crabs ( Callinectes sapidus, Rathbun). Journal of Experimental Marine Biology \& Ecology, 198(198), 155-173.

Pienaar A. G., 1990. A study of coeval sibling cannibalism in larval and juvenile fishes and its control under culture conditions. Masters thesis,Rhodes University, Grahamstown, South Africa.

Qin H., Li, Y. Q., 2014. The effects of stocking density and food on agonistic behavior and growth performance in fenneropenaeus chinensis. Oceanologia ET Limnologia Sinica, 45(4), 834-838.

Qin J. G., Mittiga L., Ottolenghi F., 2010. Cannibalism Reduction in Juvenile Barramundi Lates calcarifer by Providing Refuges and Low Light. Journal of the World Aquaculture Society, 35(1), 113-118.

Manica A., 2002. Alternative strategies for a father with a small brood: mate, cannibalise or care. Behavioral Ecology and Sociobiology, 51(4), 319-323.

Ribeiro F. F., Forsythe S., Qin, J. G., 2015. Dynamics of intracohort cannibalism and size heterogeneity in juvenile barramundi ( Lates calcarifer ) at different stocking densities and feeding frequencies. Aquaculture, 444, 55-61.

Rohwer S., 1978. Parent Cannibalism of Offspring and Egg Raiding as a Courtship Strategy. The American Naturalist, 112(984), 429-440.

Shen B. Q., Chen J. M., Ye J. Y., 2009. Effects of various substrates, stocking densities and diets on cage nursing of instar Scylla paramamosain. Journal of Zhejiang Ocean University, 28(4), 461-464.

Solomon R. J., Udoji, F. C., 2011. Canibalism Among Cultured African Catfishes (Heterbranchus Longifillis and Clarias Gariepinus). Nature \& Science.9(9):1-13.

Song G. T., Ding F. Q., Chen, J., 2012. Effects of broodstock sizes, shelter, illumination and stocking density on breeding in Red Swamp Crayfish Procambarus clarkia. Fisheries Science, 31(9), 549-553.

Song Z.Y., Wang, S. D., 2002. Set up shelter to improve the survival rate of the Portunus trituberculatus. Shandong Fisheries, 19(4), 30-31.

Sotelano M. P., Lovrich G. A., Romero M. C., Tapella F., 2012. Cannibalism during intermolt period in early stages of the Southern King Crab Lithodes santolla (Molina 1872): Effect of stage and predator-prey proportions. Journal of Experimental Marine Biology \& Ecology, 411, 52-58.

Sotelano M. P., Lovrich G. A., Tapella F., 2016. Cannibalism among Lithodes santolla, (Molina 1782) juveniles: effect of stocking density, stage and molt condition. Aquaculture International, 24(4), 1025-1037.

Triño A. T., Millamena O. M., Keenan C., 1999. Commercial evaluation of monosex pond culture of the mud crab Scylla, species at three stocking densities in the Philippines. Aquaculture, 174(1-2), 109-118.

Vallon M. Heubel, K. U., 2016. Old but gold: males preferentially cannibalize young eggs. Behavioral Ecology \& Sociobiology, 70(4), 1-5.

Verhoef G.D., Austin C. M., 1998. Combined effects of temperature and density on the growth and survival of juveniles of the Australian freshwater crayfish, Cherax destructor Clark, Part 1. Aquaculture, 170(1), 37-47.

Wang D. M., Jiang Z. Q., 2008. The factors affecting cannibalism in South flounder Paralichthys Lethostigma juveniles. Acta Hydrobiologica Sinica, 32(3), 360-364.

Wang D. M., Jiang Z. Q., 2010. Study on the factors influencing cannibalism of fish. Journal of Anhui Agri, 38(14), 7395-7397.

Wang R. J., Jiang L. X.,Li Y. Q., 2015. Effects of Internal and External Factors on Cannibalism in Post-larvae of Litopenaeus vannamei. Journal of Hebei Agricultural Sciences, 44(2), 142-145.

Wang W. J., Yang J. M., Zhou, Q. L., 2009. Observation on the cannibalistic behavior among 
Octopus ocellatus larvae. Journal of Aquaculture, 30(10), 14-18.

Wang Y.M., Zhang S.H., Zhang X.L., Qu, J. M., 2004. Application of movable and portable shelter in farming of sea cucumber. Shandong Fish, 21 (7), 9-10.

Wei H. Q., Jiang K. J., Zhang F. Y., 2015. Fight and stress behavior of aquatic animals. Fishery Information\&Strategy, 30(4), 280-287.

Wootton R. J., 1971. Measures of the Aggression of Parental Male Three-Spined Sticklebacks. Behavior, 40(3/4), 228-262.

Xi D., Zhang X., Lü, H., Zhang, Z., 2017. Cannibalism in juvenile black rockfish, Sebastes schlegelii (Hilgendorf, 1880), reared under controlled conditions. Aquaculture, 479, 682-689.

Xuan F. J., Jiang F. H., Bian, X. G., 2015. Potential of an artificial reef used for culturing of Portunus trituberculatus. Jiangsu Agricultural Science, 43(6), 206-208.

Ye Y. Z., Han Y. Z., Wang P. P., 1995. Selection of shelters for freshwater prawn and mitten crab. Fisheries Science \& Technology Information, 22(6), 243-249.

Yu W., Ma Z. H., Zhang, J. S., 2016. Cannibalism in fish and its control under artificial culture conditions. Marine Fisheries, 38(6), 689-696.

Zaret T. M., 1977. Inhibition of cannibalism in Cichla ocellaris and hypothesis of predator mimicry among South American fishes. Evolution, 31(2), 421-437.

Zeng Q. F., Erik J. E., Gu X. H., Mao, Z. G., Chen H. H., 2018. Cannibalism and Habitat Selection of Cultured Chinese Mitten Crab: Effects of Submerged Aquatic Vegetation with Different Nutritional and Refuge Values. Water, 1542(10), 2-14.

Zhang P. D., 2006. Experimental studies on the behavior and physio-ecology of penaeid shrimps. D. Ocean University of China.

Zhao Q., Zhao, Q. L., 1999. The Influence of Murdering Behavior of the Fugu Obscurus Fries on Survival Rate During the Early Growing Periods. Journal of Nan Jing Normal University, 22(4), 86-88.

Zou G. W., Lou X. Z., Pan, G. B., 2001. Studies on the cannibalism among Silurus meridionalis larvae. Journal of Fishery Sciences of China, 8(2), 55-58. 